I Congreso del Programa No Graduado, Santa Fe: otra forma de pensar y hacer la escuela. Páginas 315-318 en Revista de la Escuela de CIencias de la EduCaCión, año 11, número 10, enero a diciembre de 2015. ISSN 18516297. ISSN EN LINEA 2362-3349.

\title{
I CONGRESO DEL PROGRAMA NO GRADUADO, SANTA FE: OTRA FORMA DE PENSAR Y HACER LA ESCUELA
}

\author{
Por Dana Sokolowicz* \\ (Universidad de Buenos Aires), Argentina \\ danasoko@hotmail.com
}

Recibido: 26/06/2015 Aceptado: 02/09/2015

\section{Resumen}

La siguiente reseña presenta el I Congreso del Programa No Graduado de Santa Fe. Este programa implementa una modalidad de enseñanza diferente al aula estándar. Al desarrollar los temas tratados en el mismo, esta reseña contribuirá a enriquecer el debate sobre las condiciones pedagógicas de la escolarización hoy, al mismo tiempo que permitirá difundir prácticas pedagógicas escolares más inclusivas.

\section{Palabras clave:}

Modelos pedagógicos - Escuela no graduada - Educación inclusiva - Prácticas educativas.

\section{Abstract}

This review presents the 1st Congress of the Non-Graded Program (I Congreso del Programa No Graduado) in Santa Fe. This program implements a way of teaching that differs from standard classrooms. By explaining the topics discussed in this event, this review will contribute to enrich the debate about pedagogic conditions of current schooling. It will also contribute to spread more inclusive pedagogical practices.

Lic. en Ciencias de la Educación (UBA), Maestranda en Psicología Educacional (UBA) y Maestranda en Ciencias Sociales con Orientación en Educación (FLACSO). Profesora de las asignaturas Pedagogía I y Teorías de la Educación del Profesorado de Educación Física de la UMET y Asistente Pedagógica en el Instituto Sarmiento (Escuela de Nivel Primario, CABA). 
Revista de la Escuela de Ciencias de la Educación, año 11, número 10, enero a diciembre de 2015. Páginas 315-318. ISSN 1851-6297. ISSN en línea 2362-3349. I Congreso del Programa No Graduado, Santa Fe: otra forma de PENSAR Y HACER LA ESCUELA. DANA SOKOLOWICZ

\section{Key words:}

Pedagogic Models - Non Graded School - Inclusive Education- Educational Practices.

Una de las problemáticas actuales del campo educativo es el desgranamiento y la deserción escolar, lo que conduce a revisar algunos aspectos de los modelos pedagógicos vigentes. El congreso que aquí se reseña se inscribe en la discusión sobre las condiciones pedagógicas que adopta la escolarización en la mayoría de las escuelas actualmente.

El I Congreso del Programa No Graduado tuvo lugar los días 22 y 23 de agosto de 2014 en la ciudad de Rosario, Santa Fe y se realizó en el Club Provincial. Fue organizado por la Comisión autogestionada que coordina el programa y contó con el apoyo del Ministerio de Educación de la Provincia.

El Programa No Graduado (PNG) funciona desde hace 30 años en la Provincia de Santa Fe, en escuelas primarias públicas, tanto rurales como urbanas en la ciudad de Rosario. Este programa lleva adelante prácticas pedagógicas que sostienen una perspectiva sobre el aprendizaje y la enseñanza escolar diferente a la de la pedagogía tradicional. Se define a sí mismo como una modalidad de trabajo democrática, reflexiva, móvil y flexible, que valora los procesos de aprendizaje singulares, generando las condiciones para que cada niño/a pueda transitar su propia trayectoria escolar, entendiendo que éstas no son lineales y que todos tienen posibilidades de aprender.

Uno de los aspectos centrales del Programa es que las escuelas que participan del mismo organizan la enseñanza en grupos por niveles que reúnen a niños y niñas de distintas edades en lugar de estructurarse según los grados estándar. En concordancia con esto, otro aspecto fundamental es la mirada que tienen sobre el aprendizaje y la reflexión constante sobre las prácticas educativas, que se lleva a cabo en forma colaborativa entre los docentes de los distintos grupos, los equipos directivos y los docentes encargados de realizar un seguimiento de los procesos de aprendizaje de cada niño/a, denominados "maestros niveladores".

Del evento participaron 800 docentes, directivos y supervisores, y contó con el apoyo y presencia de Jorge Márquez (Secretario de Educación de Santa Fe) y Stella Lapelle (Directora Provincial de Educación Primaria de Santa Fe). El encuentro estuvo organizado en conferencias, espacios de debate grupal y momentos para recorrer una "Galería Pedagógica". Como expositores participaron Flavia Terigi y Guillermo Volkind, quienes con sus aportes teóricos enriquecieron la reflexión sobre las prácticas del PNG.

EI PNG es un proyecto que propone una forma diferente de pensar y organizar los aprendizajes en la escuela. Se reconoce como el eje vertebrador de las instituciones participantes y es sostenido por los docentes y equipos 
Revista de la Escuela de Ciencias de la Educación, año 11, número 10, enero a diciembre de 2015. Páginas 315-318. ISSN 1851-6297. ISSN en linea 2362-3349. I Congreso del Programa No Graduado, Santa Fe: otra forma de PENSAR Y HACER LA ESCUEla. DANA SOKOLOWICZ

de conducción de las mismas. Cada una de ellas construyó los proyectos a través de los cuales puso en práctica los principios fundamentales del PNG, entre los que se encuentran el trabajo en equipo (tanto de maestros como de alumnos), el respeto por los tiempos de aprendizaje, la movilidad pedagógica y la reflexividad.

En la mayor parte de las escuelas funciona en el primer ciclo (de $1^{\circ} \mathrm{a}$ $3^{\circ}$ grado). Los alumnos/as no se agrupan según su edad (de acuerdo con los grados estándar) sino según sus conocimientos y posibilidades. Se toma como eje la alfabetización, dado que se considera que éste no es un aprendizaje que se logra solamente en el transcurso de un año escolar ( $1^{\circ}$ grado) como se establece tradicionalmente, sino que es un proceso más largo. Por este motivo, se armaron siete niveles que los niños/as van transitando a su ritmo. Esto permite evitar la repitencia y que cada uno vaya avanzando según sus posibilidades y sus propios tiempos. Por ello, el PNG se propone como un espacio de posibilidad que permite aprovechar la flexibilidad para construir educabilidad: todos tienen capacidad de aprender.

Otro de los ejes del programa es el trabajo en equipo y en grupo. Se trabaja con parejas pedagógicas y se tiene una mirada colectiva del conjunto de los docentes acerca de cada uno de los niños/as. En este sentido, se defiende un "constructivismo democrático", que se sustenta en la participación y el compromiso de todos, en donde cada uno se compromete desde las responsabilidades de su rol.

En el espacio de conferencias, Guillermo Volkind planteó la contradicción entre la propuesta y convicción de trabajar colectivamente, y un sistema educativo que tiende a ser individualizante. Desarrolló la importancia considerar una concepción grupal del aprendizaje, en la cual éste es producto del grupo. Retomando otro de los principios clave del PNG, señaló que los aprendizajes se construyen a lo largo del tiempo, y que cada sujeto se va apropiando de los mismos a distintos ritmos. El tiempo hoy se está constituyendo en un problema. La dificultad es la tolerancia a los procesos largos. Es fundamental tener en cuenta que no hay aprendizaje si no hay tiempo para ello. Asimismo sostuvo la importancia de los talleres docentes, en los que los mismos maestros/as realicen su propio proceso de aprendizaje y reflexión conjunta. Planteó como desafío entender que el conocimiento es complejo, pero que está al alcance de todos.

En la "Galería Pedagógica" se expusieron los distintos proyectos que se llevan a cabo en las escuelas. El objetivo fue compartir y difundir los ejes articuladores de cada institución.

En función de estas reflexiones, se procedió a trabajar en grupos, espacio en el que se encontraron por primera vez distintos docentes que participan del programa, pudiendo intercambiar experiencias, dificultades, estrategias y preguntas. 
Revista de la Escuela de Ciencias de la Educación, año 11, número 10, enero a diciembre de 2015. Páginas 315-318. ISSN 1851-6297. ISSN en linea 2362-3349. I Congreso del Programa No Graduado, Santa Fe: otra forma de PENSAR Y HACER LA ESCUELA. DANA SOKOLOWICZ

Otra de las expositoras fue Flavia Terigi, quien planteó como desafío formular conocimiento pedagógico que contribuya a transformar la escuela, enfatizando la necesidad de sistematizar y difundir la experiencia del PNG. Explicó cómo este programa rompe con algunos de los supuestos pedagógico-didácticos que estructuran las prácticas escolares: la simultaneidad (todos aprenden lo mismo de la misma manera, a través de secuencias unificadas de enseñanza), la gradualidad anualizada (el avance progresivo en los conocimientos, asignando un año lectivo a grado), y las cronologías de aprendizaje unificadas (todos aprenden con los mismos tiempos, y quien no puede debe repetir el año completo). Las propuestas implementadas por el PNG contribuyen a generar prácticas y saberes sobre modelos pedagógicos alternativos que cuestionan al saber pedagógico por defecto, es decir, aquél pre-establecido y naturalizado, ampliamente difundido, que apoya el funcionamiento estándar del sistema y que nos impide pensar en direcciones novedosas.

En este sentido el PNG adquiere una relevancia especial y potente para repensar las prácticas educativas y escolares actuales. Se presenta como una alternativa que contempla otras modalidades posibles de enseñanza y aprendizaje, que puedan dar respuesta a diversas problemáticas del campo pedagógico y ofrecer una educación más inclusiva. 